Research Paper

\title{
Generation of Mesenchymal Stem Cells from Human Embryonic Stem Cells in a Complete Serum-free Condition
}

\author{
Enqin $\mathrm{Li}^{*}$, Zhenwu Zhang*, Bin Jiang, Li Yan, Jung Woo Park, Ren-He Xu ${ }^{\bowtie}$ \\ Centre of Reproduction, Development \& Aging, and Institute of Translational Medicine, Faculty of Health Sciences, University of Macau, Taipa, Macau, China \\ *These authors contributed equally. \\ $\square$ Corresponding author: Ren-He Xu, Faculty of Health Sciences, University of Macau, Taipa, Macau, China. Email address: renhexu@umac.mo. Phone: +853 \\ $8822-4993$ \\ (c) Ivyspring International Publisher. This is an open access article distributed under the terms of the Creative Commons Attribution (CC BY-NC) license \\ (https://creativecommons.org/licenses/by-nc/4.0/). See http://ivyspring.com/terms for full terms and conditions.
}

Received: 2018.02.02; Accepted: 2018.08.11; Published: 2018.10.23

\begin{abstract}
Mesenchymal stem cells (MSC) have been derived from a variety of tissues, and cultured either in animal serum-containing (SC) or serum-free (SF) media. We have previously derived MSC from human embryonic stem cells via an intermediate trophoblast step (named EMSC), which also have immunosuppressive and therapeutic effects on animal models of autoimmune disease. To promote the clinical application of this new source of MSC, we report here EMSC derived and cultured in a SF medium MesenCult (SF-EMSC) in comparison with a SC medium (SC-EMSC). SF-EMSC derived in MesenCult also expressed typical MSC markers CD73, CD90, and CD105, and manifested multipotency to differentiate to osteocytes, chondrocytes, and adipocytes. Comparably, $\mathrm{CD} 105^{+}$ cells reached $90 \%$ about one week slower in the SF than SC conditions, and the proliferation rate was slightly faster for SF-EMSC than SC-EMSC at later passages. Both SF- and SC-EMSC responded similarly to the inflammatory stimulus IFNy. However, the inflammatory cytokines IL- 6 and IL-8 were expressed much less in SF-EMSC than SC-EMSC. Furthermore, knockdown of P16 ${ }^{\text {INK4A }}$ in both SF- and SC-EMSC reduced replicative senescence. Together, our results suggest that EMSC can be generated in a complete SF condition, and SF-EMSC are largely similar to SC-EMSC. However, it takes longer time to derive EMSC in the SF than SC conditions, and the SF-EMSC proliferate faster at later passages and produce less of the inflammatory cytokines IL-6 and IL-8 than SC-EMSC. This study provides important information for production of clinically applicable EMSC.
\end{abstract}

Key words: Serum, mesenchymal stem cells, human embryonic stem cells, derivation, and culture

\section{Introduction}

Mesenchymal stem cells (MSC) are a population of stromal cells with the capability of self-renewal and multipotent differentiation $[1,2]$. MSC are present in most, if not all, tissue types, and have been isolated from a variety of tissues such as amniotic membrane, umbilical cord, bone marrow and adipose [2]. MSC can also be derived from pluripotent stem cells (PSC) including embryonic stem (ES) and induced pluripotent stem (iPS) cells [3-9]. In addition to their potential for replacing damaged and diseased tissues by differentiating into tissue-specific cells or providing reparative and nutrient factors, human PSC-derived MSC have been shown to modulate functions of immune cells and exert diseasemodifying efficacy in animal models of many autoimmune and inflammatory diseases [9-12]. MSC-based clinical trials have been ongoing worldwide although the efficacy of MSC has not yet been conclusive.

Many culture media have been used for MSC isolation and expansion, and can be categorized into two types, i.e., serum-containing (SC) and serum-free 
(SF). Almost all sera used for mammalian cell culture are derived from animals. Xenogeneic and undefined components contained in animal sera cause not only complexity and uncertainty when used for studying MSC, but also safety concerns for their clinical application. To overcome this problem, many SF media have been developed. Among them, MesenCult medium is not only SF, but also xeno-free (free of animal products) and contains only defined components.

The methods for MSC derivation from human ES cells (hESC) or iPS cells include direct differentiation, embryoid body formation, co-culturing with mouse OP9 cells, sorting, scraping or handpicking of cells [3-8]. Although various advantages are associated with some of the methods, they are all limited by low efficiency and tedious procedures, and all depend on use of SC media. We have recently found that MSC can be derived via an intermediate step - trophoblast formation (named EMSC) in monolayer [9] or a 3-dimentional method starting from hESC spheres [13]. The first step of EMSC derivation (trophoblast differentiation from hESC) is induced by BMP4 and A83-01 (an inhibitor of TGF $\beta$ signaling) in the commercially available SF medium, mTeSR Minus Select Factors, in which lithium chloride, bFGF, and TGF $\beta$ were removed $[14,15]$, however the second step (MSC differentiation from the trophoblasts) has to be completed in a SC medium (called MSC medium).

Replicative senescence is an irreversible process that leads to the loss of proliferative potential and permanent growth arrest of any differentiated or partially differentiated cells including MSC [16]. It has been well known that P16 INK4A (P16) plays an important role in the regulation of proliferation and senescence of human MSC. More senescent cells and higher expression of $\mathrm{P} 16^{\mathrm{INK} 4 \mathrm{~A}}$ have been found in human bone marrow MSC (BM-MSC) at high passages than low passages, and inhibition of P16 delays senescence of human BM-MSC [17].

This study was attempted to remove this hurdle by generating EMSC in a completely SF medium such as MesenCult. We found that MesenCult supported the derivation and proliferation of EMSC. EMSC derived in the SF medium (SF-EMSC) used longer time for derivation, proliferated faster after derivation, and expressed less the inflammatory cytokines IL-6 and IL-8 than EMSC derived in the serum-containing (SC) condition (SC-EMSC). P16 was involved in the proliferation and senescence of both SF- and SC-EMSC.

\section{Results}

\section{Generation of EMSC in SF media}

Human ESC lines H9 and CT3 were used in this study [18]. SF- and SC-EMSC were derived from hESC as shown in a schematic (Fig. 1A). hESC were routinely propagated in E8 (Stemcell Technologies), which is a SF, xeno-free, albumin-free, and all-defined medium [19]. We first split hESC with small colonies and cultured the cells in E8. On day 2-3, consumed medium was removed and cells were induced to differentiate into trophoblasts via treatment with 10-ng/ml BMP4 and 1- $\mu$ M A83-01 in E6, which lacked FGF2 and TGF $\beta 1$ compared to E8, as we reported previously except that mTeSR Minus Select Factors was replaced with E6 [9]. Within 3-5 days, hESC uniformly became flattened and acquired trophoblastlike cell morphology and positive for the trophoblast marker TROP2, while untreated hESC remaining positive for the pluripotency marker OCT4 (Fig. 1B).

On day 5, further differentiation from trophoblast-like cells to MSC was initiated by replacing the spent medium with either MesenCult (SF) or the MSC medium (SC), i.e., $\alpha \mathrm{MEM}$ supplemented with $20 \%$ fetal bovine serum and $1-\mathrm{mM}$ L-glutamine. The medium was refreshed every 2-3 days and mesenchymal-like cells emerged in 1-2 and 2-3 weeks in the SC and SF medium, respectively. By day 19 (in SC) or 35 (in SF), over $90 \%$ of the cells were stained positive for the MSC marker CD44, CD73, CD90, and CD105 (with only CD44 shown in Fig. 1B), which was confirmed via flow cytometry (Fig. 1C). However, the cells were negative for a cocktail of hematopoietic markers (Fig. 1C). The mesenchymallike cells were split weekly and finally became MSC with homogeneous phenotypes within 3-4 (for SC) or 4-5 (for SF) weeks, and are named SC- and SF-EMSC, respectively (Fig. 1A). CD105 appeared slower during generation of SF-EMSC than SC-EMSC, especially at the early times (Fig. 1D). MesenCult and MSC medium were continuously used to maintain SF- and SC-EMSC, respectively.

To simplify the differentiation system, we also tried to use MesenCult, instead of E6, to differentiate hESC into trophoblasts in the presence of BMP4 and A83-01. However, cells in this condition quickly detached and died. Thus, MesenCult could not replace E6 in the initial step of the MSC derivation. Furthermore, we confirmed the multipotency of SFand SC-EMSC continuously cultured in the corresponding medium and found both could differentiate into osteocytes, chondrocytes or adipocytes within two weeks or so (Fig. 1E). Therefore, both SC- and SF-EMSC meet the minimal standards for MSC [20]. EMSC were also derived from another hESC line CT3 in either SF- or SC condition, and expressed the typical MSC markers and demonstrated the capability of the tri-lineage differentiation (Fig. S1). 


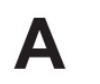




\section{Proliferation of EMSC in the SF- and SC-media}

Next, we evaluated the proliferation rate of both SF- and SC-EMSC by detecting Ki67, a marker for cell division, by immunostaining. As shown in Fig. 2A, the ratio of $\mathrm{Ki}^{+} 7^{+}$cells were $49.1 \pm 2.2 \%$ (p3) and $38.0 \pm 1.3 \%$ (p7) for SF-EMSC and $51.5 \pm 2.6 \%$ (p3) and $26.4 \pm 1.4 \%$ (p7) for SC-EMSC, respectively. The proliferation capacity of SF- and SC-EMSC was similar to each other at earlier passage (p3). However, the proliferation of SC-EMSC started to slow down at p7, while SF-EMSC still kept high proliferation capacity. SF- and SC-EMSC could continuously expand in the corresponding medium for up to 12 passages exponentially with accumulated cell number increased about 1,012- and 1,010-fold in the SF and SC groups, respectively (Fig. 2B). QPCR results showed that the expression of cell cycle related genes (CCND1, $M C M 7, C C N D 3$ and $C C N B 2)$ and DNA replication related genes (POLA1 and POLE2) were higher in SF-EMSC than SC-EMSC (Fig. 2C). Thus, SF-EMSC proliferated faster than SC-EMSC in later passage. After p15 or so, the proliferation of both SF- and SC-EMSC remarkably declined (data not shown).

\section{Replicative senescence of SF-EMSC}

Since replicative senescence develops during passaging of MSC, we compared the development of replicative senescence in both SF- and SC-EMSC. We found that $P 16$ expression was higher in both SF- and SC-EMSC at a high passage (p9) than a low passage (p2) (Fig. 3A). Therefore, we decided to test whether knockdown of P16 can rescue EMSC from senescence. We established stable SC- and SF-EMSC lines with P16 knockdown using a lentiviral vector to co-express green fluorescent protein (GFP) and P16 shRNA (shP16) or a scrambled shRNA as a negative control (shNC). $\mathrm{GFP}^{+}$cells were sorted and used for the following analyses. P16 expression was remarkably reduced in both SC-EMSC and SF-EMSC with shP16 than shNC as detected via RT-PCR (Fig. 3B), and confirmed via Western blotting (Fig. 3C). On the other hand, senescence-associated $\beta$-galactosidase (SA- $\beta$ gal) staining showed that P16 knockdown reduced cellular senescence (Fig. 3D) and promoted proliferation (Fig.3E) of both SC-EMSC and SF-EMSC.

A

P3

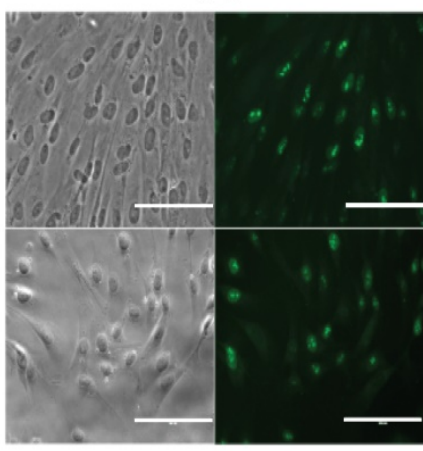

B

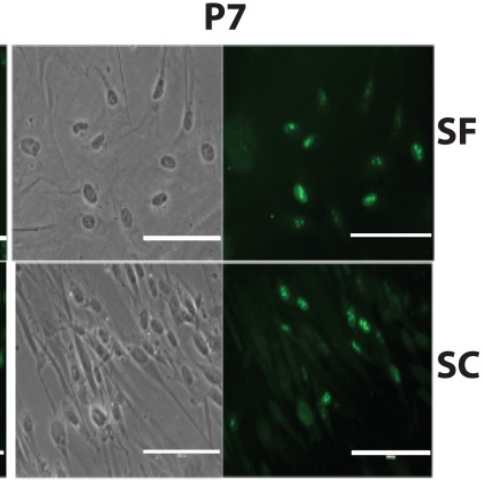

SF-EMSC

$\rightarrow$ SC-EMSC

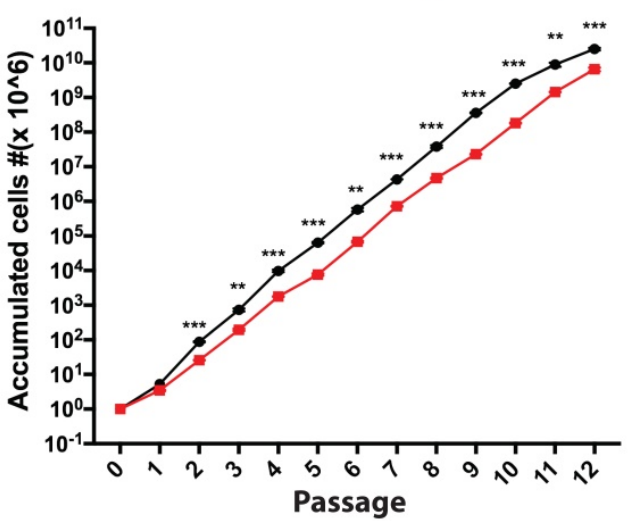

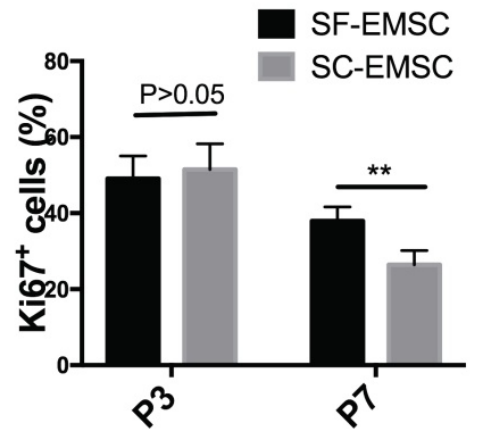

C

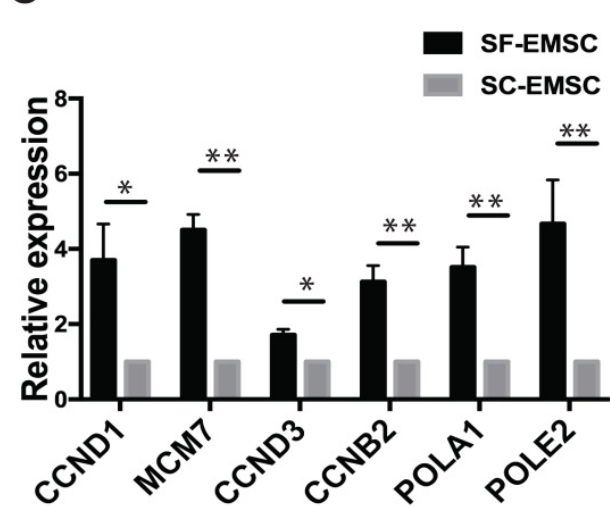

Figure 2. Analyses of proliferation of SF- and SC-EMSC at various passages. (A) Immunofluorescent staining analyzed Ki67 expression in both SF- and SC-EMSC at passage 3 and 7 . Bars, $100 \mu \mathrm{m}$. $* * P<0.01$. (B) The total number of cells recovered at each subsequent passage was calculated and plotted. $* * P<0.01$, $* * * P<0.001$. (C) The expression of cell cycle related genes and DNA replication related genes were analyzed by $q P C R$. Gene expression was normalized to the value for GAPDH. 


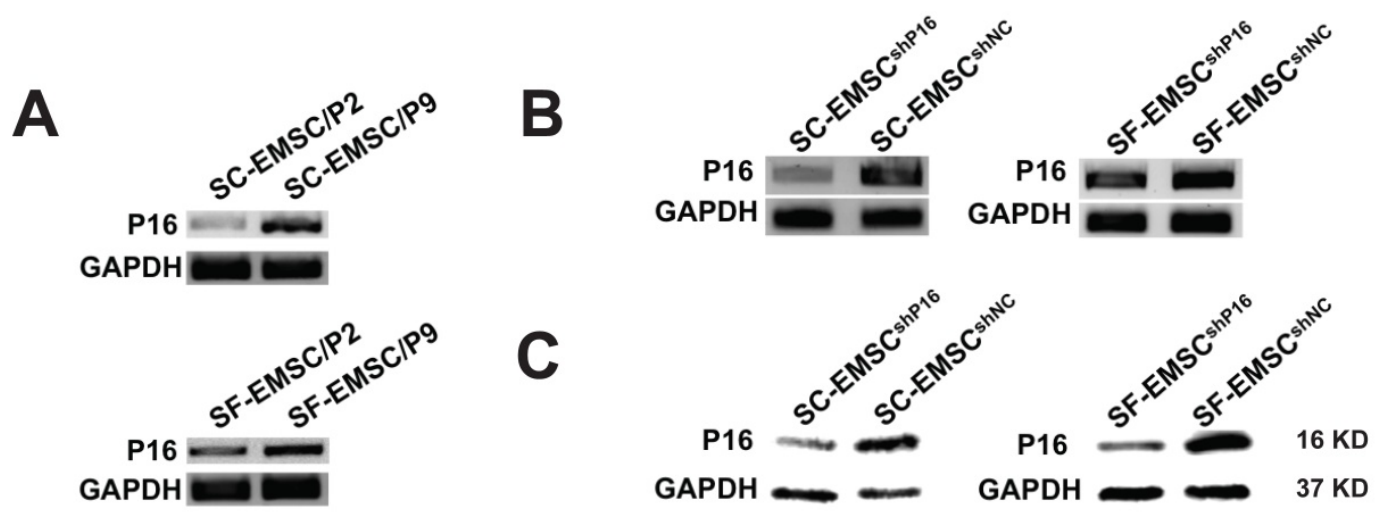

D

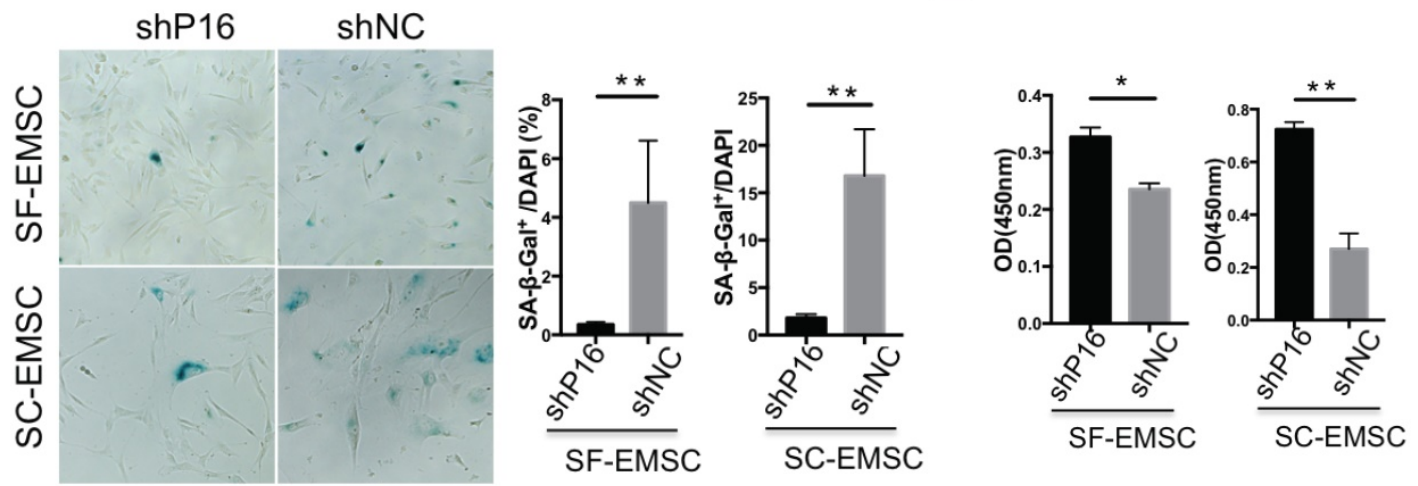

Figure 3. Knockdown of P16 rescued SF- and SC-EMSC from senescence. (A) P16 mRNA expression in an indicated passage of SF- and SC-EMSC was detected by RT-PCR. GAPDH was used as a loading control. (B) SF- and SC-EMSC were transduced with lentivirus co-expressing GFP and shP16 or shNC, GFP+ cells were sorted and tested for PI 6 expression via RT-PCR. GAPDH was used as a loading control. (C) P16 protein in GFP+ SF- and SC-EMSC was detected via western blotting. GAPDH was used as a loading control. (D) The senescence of GFP+ SF- and SC-EMSC were detected by senescence-associated beta-galactosidase assays. Total cell number was counted by DAPI staining. (E) Cell proliferation was evaluated GFP+ SF- and SC-EMSC using the CCK-8 assay. $* P<0.05, * * P<0.01$.

\section{Immunomodulatory cytokines generated by SF- and SC-EMSC}

Cytokines including interferons, tumor necrosis factor, and interleukins play critical role in mediating MSC effects [21]. We have previously observed that MSC derived from hESC via other methods had higher secretion of the anti-inflammatory cytokine IL-8 and lower secretion of the pro-inflammatory cytokine IL-6 than BM-MSC [9, 22]. Using RT-PCR (Fig. 4A) and cytokine bead array (CBA) (Fig. 4B), we found here that the basal levels of both IL- 6 and IL-8 secreted by SF-EMSC were much lower than SC-EMSC. It has been known that priming of MSC with pro-inflammatory cytokines such as IFN $\gamma$ can change the profile of cytokine expression and enhance the immunosuppressive effects of the cells [23]. Upon stimulation with IFN $\gamma$, the expression of IL-6 was increased and IL-8 was decreased in BM-MSC; IL-6 and IL-8 were not changed in SF-EMSC; IL-8 was reduced in SC-EMSC but IL-6 was not changed. It has been reported that immunosuppression by human- or monkey-derived MSC is mediated by indoleamine 2,3-dioxygenase (INDO or IDO) and PDL1 [24]. IDO (Fig. 4C) and PDL1 (Fig. 4D) expression dramatically increased in both SC- and SF-EMSC following IFN $\gamma$ treatment. These data suggest that both SC- and SF-EMSC can generate immunomodulatory cytokines in response to the potent immunological stimulus IFN $\gamma$.

\section{Discussion}

MSC derived from hPSC share great similarities with MSC obtained from other sources. As established pluripotent cell lines, hPSC also have unique advantages. They are an unlimited source for generation of MSC and many other cell types, easy for quality control for pathogen-free and standardized production, and appropriate for genetic manipulation, for example, to reduce immunogenicity and tumorigenicity [25]. As a result, hPSC-derived MSC are potentially a better candidate than other sources for clinical applications to treat many degenerative and autoimmune diseases. However, in order to use these cells in clinical settings, it is mandatory to establish a consistently reproducible 
protocol for large-scale production and define a standard operating procedure compliable with good manufacturing practice (GMP) including selection of an optimal cell therapy-compliant culture medium. We have recently reported that MSC can be derived from hESC via a trophoblast-like stage and expanded to large scale in a reasonable time without altering their basic characteristics [9]. Although the differentiation into trophoblasts was conducted in a SF medium, the subsequent differentiation into MSC and expansion of the resultant MSC were in a SC medium, thus not ideal for GMP-compliable generation of clinical-grade of MSC.

Here we have attempted to develop a completely SF system to generate and expand EMSC. We first differentiated hESC into trophoblast-like cells in the SF medium E6 supplemented with BMP4 and A83-01, and then differentiated the trophoblast-like cells into MSC in another SF medium MesenCult, then maintained and expanded the SF-EMSC in MesenCult. It took a bit longer time (4-7 days) to establish SF-EMSC than SC-EMSC due to slower appearance of CD105. SF- and SC-EMSC proliferated at similar rates up to 15 passages and then SF-EMSC proliferated slightly faster than SC-EMSC. Knockdown of P16 ${ }^{\mathrm{INK}} 4 \mathrm{~A}$ rescued senescence and promoted proliferation in both SF-EMSC and SC-EMSC. Similar results were observed with another SF medium StemPro (data not shown).

MesenCult was designated to help MSC researchers to standardize their culture systems and

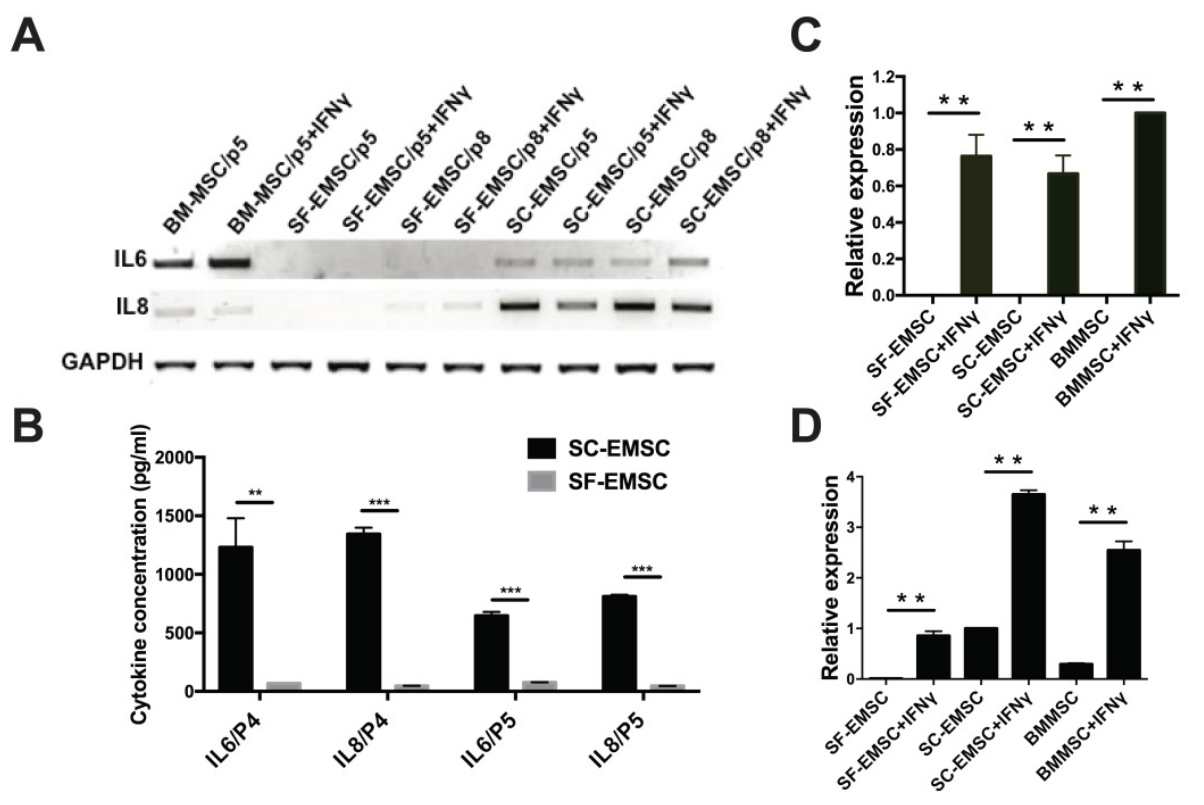

Figure 4. Comparison of inflammatory cytokine expression between SF- and SC-EMSC. (A) Gene expression of IL-6 and IL8 in SF- and SC-EMSC were detected by RT-PCR. GAPDH was used as loading control. (B) IL-6 and IL-8 protein were analyzed by Cytometric Bead Array. (C and D) The expression of IDOI (C) and PDLI (D) in EMSC with or without IFN $\gamma$ treatment was detected via qPCR. Gene expression was normalized to GAPDH. $* * p<0.01$, **** $<0.001$. reduce variability of MSC cultures. Although it was originally developed for isolation and expansion of human BM-MSC [26], it has also been used for isolation and expansion of MSC from many other sources such as human umbilical cord blood [27, 28], Wharton's jelly [29], adipose [30], corneal limbus [31], and even teratomas formed by hESC [32]. The cell morphology, proliferation rate, senescence development, differentiation capability, immunosuppressive effect, cytogenetic stability, tumorigenicity, etc., of MSC cultured in different media have been carefully analyzed. Given the general recognition of the capability of MesenCult (as well as some other SF media) to support isolation and expansion of MSC from various sources, controversial observations have been reported between MSC cultured in the SF media versus conventional SC media.

For example, MSC derived from bone marrow [26], umbilical cord blood (UCB) [27], Wharton's jelly [29], and adipose [30] all have higher proliferation rate and yield in MesenCult than SC media. However, Gottipamula, et al., [33] found that BM-MSC cultured in SF media including MesenCult and the BD Mosaic Mesenchymal Stem Cell Serum-Free Medium (BD-SFM) grow much slower than those in a control SC medium. Comparably, we found that EMSC had similar and steady proliferation up to p15 in both the SC and SF media (faster in the SF medium than the SC medium at later passage), with a short PD time at around $20 \mathrm{~h}$ (Fig. 2B). Post-thaw viability of BM-MSC derived from both the SF and SC media were found to be above $90 \%$ [33]. We also observed over $90 \%$ post-thaw viability of EMSC that had been cultured in either the SF or SC medium and frozen at various passages (data not shown).

Furthermore, IFN- $\gamma$ treatment increases the expression level of the immunosuppressive molecule IDO in BM-MSC cultured in either the SC or SF conditions [33], which is consistent with our observations with EMSC.

In summary, we have demonstrated an array of common and different features between EMSC derived and maintained in SC and SF conditions. Both conditions allowed derivation of EMSC. However, it took a bit longer time to derive 
SF-EMSC than SC-EMSC, and SF-EMSC proliferated faster at later passages and produced less of the inflammatory cytokines IL-6 and IL-8 than SC-EMSC. These differences might be relevant to differential expression of some genes essential for development of inflammation, senescence and immunosuppression between SF- and SC-EMSC. The exact mechanism regarding the culture-related differences awaits further studies.

\section{Methods}

\section{Culture of hESC and Generation of EMSC in SF media}

Under approval of the Research Ethics Panel of University of Macau, hESC lines H9 and CT3 were used in this study. hESC were split into a 6 well plate coated with Matrigel (BD Bioscience) at about $5 \times 10^{4}$ cells/well in E8 medium [19] (Stemcell Technologies) at $37{ }^{\circ} \mathrm{C}$ and $5 \% \mathrm{CO}_{2}$, and cultured overnight. For trophoblast differentiation, the spent media was removed, each well was washed with PBS twice, and E6 (E8 minus FGF2 and TGF 31 ) (Stemcell Technologies) was added at $2 \mathrm{ml} /$ well together with $10 \mathrm{ng} / \mathrm{ml} \mathrm{BMP} 4$ and $1 \mu \mathrm{M}$ A83-01 (Stemgent). Cells were cultured at $37{ }^{\circ} \mathrm{C}$ and $5 \% \mathrm{CO}_{2}$, and monitored under microscope every day.

At day 3-5, cells were washed with PBS. Without passaging, the cells were directly cultured in MesenCult (Stemcell Technologies) or MSC medium, i.e., $\alpha \mathrm{MEM}$ medium supplemented with $20 \%$ fetal bovine serum (GIBCO), L-glutamine (GIBCO) and $1 x$ non-essential amino acids (GIBCO) at $2 \mathrm{ml} /$ well. The medium was refreshed every two days and the cells were checked for expression of the MSC markers CD73, CD44, CD90, and CD105 periodically. When over $90 \%$ of the cells became positive for all the four markers, we designated the cells as passage-1 EMSC. The cells were split when they reached 80-90\% confluence in each passage, which took about every 5-7 days.

\section{Flow cytometry for cell surface markers}

Flow cytometry staining was performed using standard methods and antibodies against TROP2, CD31, CD34, CD73, CD90, CD105, and CD44 (BD Bioscience). Data were collected on Accuri C6 flow cytometer (BD Bioscience). Data analysis was performed with Accuri C6 software.

\section{Immunocytochemistry}

Immunocytochemistry was performed as described before [34] using anti-OCT4 (sc-5279, Santa Cruz Biotechnology) and anti-TROP2 (BD Bioscience) antibodies. Cell nuclei were counterstained with DAPI.

\section{Tri-lineage Differentiation of EMSC}

EMSC were differentiated into osteogenic, adipogenic and chondrogenic lineages using StemPro differentiation kits (Life Technologies), according to the manufacturer's protocols.

\section{Cytometric Bead Array (CBA)}

Spent medium from cultures of EMSC with or without IFN $\gamma$ treatment was collected and subjected to CBA assay to analyze levels of multiple cytokines in the medium using CBA Flex Sets (BD Bioscience), according to the manufacturer's instructions. The concentration of each cytokine was calculated based on standard curves generated from serial dilutions of cytokine standards provided by the manufacturer.

\section{$\boldsymbol{\beta}$-Galactosidase staining for senescent cells}

The Senescence $\beta$-Galactosidase Staining Kit (Cell Signaling Technology) was used according to the manufacturer's instructions. Briefly, following removal of culture medium, cells were rinsed one time with PBS, and fixed with 1x Fixative Solution (1 $\mathrm{ml} /$ well) for 10-15 $\mathrm{min}$ at room temperature. Following removal of the Fixative Solution, cells were rinsed twice with PBS, and treated with the $\beta$-Galactosidase Staining Solution $(2 \mathrm{ml} /$ well). The plate was sealed with parafilm to prevent evaporation, and incubated at $37^{\circ} \mathrm{C}$ overnight in a dry incubator (without $\mathrm{CO}_{2}$ ). While the $\beta$-Galactosidase Staining Solution was still on the plate, the cells were checked under a microscope for the development of blue color. Cell nuclei were counterstained with DAPI.

\section{RT-PCR and qPCR}

RNA was isolated from cells using TRIzol reagent (Invitrogen), and cDNA was synthesized from RNA using Superscript II (Invitrogen), according to the manufacturer's instructions. Gene expression was assessed through PCR and qPCR with primers for specific genes (Table S1). QPCR was performed using the CFX96 Real-Time System (BioRad). The relative amount of each mRNA to GAPDH was calculated using the $2^{-\Delta \Delta C t}$ method.

\section{shRNA-mediated P16 Knockdown}

To generate the pLVTHM-shP16 vector, we cloned oligonucleotides sequences targeting ACC AGA GGC AGT AAC CAT G into the MluI and ClaI sites of the lentiviral vector pLVTHM that expressed GFP. As a negative control, the pLVTHM-shNC vector was generated using a scramble sequence CGG AGG CTT ACA GTC TGG T cloned into the MluI and ClaI sites of pLVTHM. Either pLVTHM-shP16 or pLVTHM-shNC was co-transfected together with 
Delta R 8.74 and pMDG-VSVG to 293T cells with X-tremeGENE HP DNA Transfection Reagent (Roche) to package the lentiviruses. The lentiviruses were then transduced into SC-EMSC or SF-EMSC. GFP ${ }^{+}$positive cells were sorted via flow cytometry BD FACSARIA III (BD Bioscience) to establish stably transduced EMSC lines.

\section{Western blot}

Cellular total proteins were extracted using RIPA buffer supplemented with proteinase inhibitor (Thermo Fisher Scientific). Proteins were separated on 12\% SDS-PAGE gels and electroblotted onto PVDF membrane. The membranes were incubated with antibodies against P16 (BD Bioscience) and GAPDH (SANTA CRUZ). The membranes were then blotted with HRP-conjugated secondary antibodies.

\section{CCK-8 assay}

For CCK-8 assay, SF-EMSC or SC-EMSC was plated into 96-well plates. Three days later, the cells were incubated in a CCK-8 solution for $2 \mathrm{~h}$. The amount of formazan dye was measured using absorbance at $450 \mathrm{~nm}$ with a microplate reader.

\section{Statistical analyses}

Data are presented as mean \pm SD. Student's $t$ test was used for statistical analysis. Percentage data was arcsine transformed prior to analysis. $P<0.05$ was considered significant, and $P<0.01$ highly significant.

\section{Supplementary Material}

Supplementary figures and tables.

http://www.ijbs.com/v14p1901s1.pdf

\section{Acknowledgments}

This work was supported by University of Macau Research Committee funds MYRG \#2015-00169-FHS, \#2016-00070-FHS, and \#201700124-FHS, and Macau Science and Technology Development Fund (FDCT) \#028/2015/A1 and \#095/2017/A1 to R.X.

\section{Author contributions}

E.L., and R.-H.X. conceived and designed the research. E.L., Z.Z., B.J., and L.Y. performed the experiments. E.L., Z.Z., and R.-H.X. analyzed the data and wrote the manuscript. J.P. helped revise the manuscript. R.X gave the final approval of the manuscript.

\section{Competing Interests}

R.X. is a scientific advisor to ImStem Biotechnology, Inc., a stem cell company. The above listed authors declare no competing financial interests.

\section{References}

1. Friedenstein AJ, Chailakhjan RK, Lalykina KS. The development of fibroblast colonies in monolayer cultures of guinea-pig bone marrow and spleen cells. Cell Tissue Kinet. 1970; 3: 393-403.

2. Pittenger MF, Mackay AM, Beck SC, Jaiswal RK, Douglas R, Mosca JD, et al. Multilineage potential of adult human mesenchymal stem cells. Science. 1999; 284: 143-7.

3. Barberi T, Willis LM, Socci ND, Studer L. Derivation of multipotent mesenchymal precursors from human embryonic stem cells. PLoS Med. 2005; 2: $\mathrm{e} 161$.

4. Olivier EN, Rybicki AC, Bouhassira EE. Differentiation of human embryonic stem cells into bipotent mesenchymal stem cells. Stem Cells. 2006; 24: 1914-22.

5. Brown SE, Tong W, Krebsbach PH. The derivation of mesenchymal stem cells from human embryonic stem cells. Cells Tissues Organs. 2009; 189: 256-60.

6. Hwang NS, Varghese S, Lee HJ, Zhang Z, Ye Z, Bae J, et al. In vivo commitment and functional tissue regeneration using human embryonic stem cell-derived mesenchymal cells. Proc Natl Acad Sci U S A. 2008; 105: 20641-6.

7. Gruenloh W, Kambal A, Sondergaard C, McGee J, Nacey C, Kalomoiris S, et al. Characterization and in vivo testing of mesenchymal stem cells derived from human embryonic stem cells. Tissue Eng Part A. 2011; 17: 1517-25.

8. Vodyanik MA, Yu J, Zhang X, Tian S, Stewart R, Thomson JA, et al. A mesoderm-derived precursor for mesenchymal stem and endothelial cells. Cell stem cell. 2010; 7: 718-29.

9. Wang X, Lazorchak AS, Song L, Li E, Zhang Z, Jiang B, et al. Immune modulatory mesenchymal stem cells derived from human embryonic stem cells through a trophoblast-like stage. Stem Cells. 2016; 34: 380-91.

10. Kimbrel EA, Kouris NA, Yavanian GJ, Chu J, Qin Y, Chan A, et al. Mesenchymal stem cell population derived from human pluripotent stem cells displays potent immunomodulatory and therapeutic properties. Stem Cells Dev. 2014; 23: 1611-24.

11. Yan L, Jiang B, Niu Y, Wang H, Li E, Yan Y, et al. Intrathecal delivery of human ESC-derived mesenchymal stem cell spheres promotes recovery of a primate multiple sclerosis model. Cell Death Discovery. In press.

12. Liao H, Wang H, Rong X, Li E, Xu RH, Peng Y. Mesenchymal Stem Cells Attenuate Radiation-Induced Brain Injury by Inhibiting Microglia Pyroptosis. Biomed Res Int. 2017; 2017: 1948985.

13. Yan L, Jiang B, Li E, Wang X, Ling Q, Zheng D, et al. Scalable Generation of Mesenchymal Stem Cells from Human Embryonic Stem Cells in 3D. Int J Biol Sci. 2018; 14: 5.

14. Xu RH, Chen X, Li DS, Li R, Addicks GC, Glennon C, et al. BMP4 initiates human embryonic stem cell differentiation to trophoblast. Nature biotechnology. 2002; 20: 1261-4.

15. Wu Z, Zhang W, Chen G, Cheng L, Liao J, Jia N, et al. Combinatorial signals of activin/nodal and bone morphogenic protein regulate the early lineage segregation of human embryonic stem cells. The Journal of biological chemistry. 2008; 283: 24991-5002.

16. Shibata KR, Aoyama T, Shima Y, Fukiage K, Otsuka S, Furu M, et al. Expression of the p16INK4A gene is associated closely with senescence of human mesenchymal stem cells and is potentially silenced by DNA methylation during in vitro expansion. Stem Cells. 2007; 25: 2371-82.

17. Shibata KR, Aoyama T, Shima Y, Fukiage K, Otsuka S, Furu M, et al. Expression of the p161NK4A gene is associated closely with senescence of human mesenchymal stem cells and is potentially silenced by DNA methylation during in vitro expansion. Stem cells. 2007; 25: 2371-82.

18. Lin G, Martins-Taylor K, Xu RH. Human embryonic stem cell derivation, maintenance, and differentiation to trophoblast. Methods in molecular biology. 2010; 636: 1-24.

19. Chen G, Gulbranson DR, Hou Z, Bolin JM, Ruotti V, Probasco MD, et al. Chemically defined conditions for human iPSC derivation and culture. Nature methods. 2011; 8: 424-9.

20. Dominici M, Le Blanc K, Mueller I, Slaper-Cortenbach I, Marini F, Krause D, et al. Minimal criteria for defining multipotent mesenchymal stromal cells. The International Society for Cellular Therapy position statement. Cytotherapy. 2006; 8: 315-7.

21. Yan L, Zheng D, Xu RH. Critical Role of Tumor Necrosis Factor Signaling in Mesenchymal Stem Cell-Based Therapy for Autoimmune and Inflammatory Diseases. Front Immunol. 2018; 9: 1658.

22. Wang X, Kimbrel EA, Ijichi K, Paul D, Lazorchak AS, Chu J, et al. Human ESC-derived MSCs outperform bone marrow MSCs in the treatment of an EAE model of multiple sclerosis. Stem Cell Reports. 2014; 3: 115-30.

23. Shi Y, Su J, Roberts AI, Shou P, Rabson AB, Ren G. How mesenchymal stem cells interact with tissue immune responses. Trends Immunol. 2012; 33: 136-43.

24. Ren G, Su J, Zhang L, Zhao X, Ling W, L'Huillie A, et al. Species variation in the mechanisms of mesenchymal stem cell-mediated immunosuppression. Stem Cells. 2009; 27: 1954-62.

25. Zheng D, Wang $\mathrm{X}, \mathrm{Xu}$ RH. Concise Review: One Stone for Multiple Birds: Generating Universally Compatible Human Embryonic Stem Cells. Stem Cells. 2016; 34: 2269-75 
26. Miwa H, Hashimoto $Y$, Tensho K, Wakitani S, Takagi M. Xeno-free proliferation of human bone marrow mesenchymal stem cells. Cytotechnology. 2012; 64: 301-8.

27. Hussain I, Magd SA, Eremin O, El-Sheemy M. New approach to isolate mesenchymal stem cell (MSC) from human umbilical cord blood. Cell Biol Int. 2012; 36: 595-600.

28. Vasaghi A, Dehghani A, Khademalhosseini Z, Khosravi Maharlooei M, Monabati A, Attar A. Parameters that influence the isolation of multipotent mesenchymal stromal cells from human umbilical cord blood. Hematol Oncol Stem Cell Ther. 2013; 6: 1-8

29. Swamynathan $P$, Venugopal $P$, Kannan S, Thej C, Kolkundar U, Bhagwat S, et al. Are serum-free and xeno-free culture conditions ideal for large scale clinical grade expansion of Wharton's jelly derived mesenchymal stem cells? A comparative study. Stem cell research \& therapy. 2014; 5: 88 .

30. Al-Saqi SH, Saliem M, Asikainen S, Quezada HC, Ekblad A, Hovatta O, et al. Defined serum-free media for in vitro expansion of adipose-derived mesenchymal stem cells. Cytotherapy. 2014; 16: 915-26.

31. Bray LJ, Heazlewood CF, Munster DJ, Hutmacher DW, Atkinson K, Harkin DG. Immunosuppressive properties of mesenchymal stromal cell cultures derived from the limbus of human and rabbit corneas. Cytotherapy. 2014; 16: 64-73

32. An SM, Zeng Q, Teng XY, Long ZG, Li J, Pan Q, et al. [Growing of human embryonic stem cells on feeders derived from themselves]. Yi Chuan. 2008; 30: $1567-73$

33. Gottipamula S, Ashwin KM, Muttigi MS, Kannan S, Kolkundkar U, Seetharam $\mathrm{RN}$. Isolation, expansion and characterization of bone marrow-derived mesenchymal stromal cells in serum-free conditions. Cell Tissue Res. 2014; 356: 123-35

34. Zeng H, Guo M, Martins-Taylor K, Wang X, Zhang Z, Park JW, et al. Specification of region-specific neurons including forebrain glutamatergic neurons from human induced pluripotent stem cells. PLoS ONE. 2010; 5: e11853. 